\title{
LAS LOMBRICES DE TIERRA Y LOS MICROORGANISMOS: DESENTRAÑANDO LA CAJA NEGRA DEL VERMICOMPOSTAJE
}

\author{
Manuel Aira \& Jorge Domínguez \\ Departamento de Ecología y Biología Animal, Facultad de Biología, Universidad de Vigo, E-36310 \\ Vigo.Spain,Email: aira@uvigo.es,jdguez@uvigo.es
}

\begin{abstract}
Aira, M. \& J. Domínguez. 2010. Las lombrices de tierra y los microorganismos: desentrañando la caja negra del vermicompostaje. Acta Zoológica Mexicana (n.s.), Número Especial 2: 385-395.

RESUMEN. Los efectos de las lombrices sobre los microorganismos son directos (incremento o descenso de sus poblaciones al digerir el sustrato) e indirectos (efectos derivados de los directos, como la aparición de las deyecciones en el sustrato fresco). Para delimitar y poder estudiar estos efectos por separado realizamos tres experimentos. En el primero estudiamos el proceso de vermicompostaje con Eisenia fetida de purín de cerdo en todas sus etapas, desde el residuo fresco hasta obtener el vermicompost. En el segundo inoculamos vermicompost en purín de cerdo fresco para estudiar los efectos indirectos de las lombrices de tierra en la degradación del purín. En el tercero analizamos las deyecciones frescas para estudiar los efectos directos de las lombrices de tierra en los microorganismos. Las lombrices de tierra dividieron el proceso de vermicompostaje en dos etapas, una inicial, asociada a su presencia en el residuo, caracterizada por el aumento de la biomasa y actividad de los microorganismos; y la etapa final, asociada a la ausencia de las lombrices de tierra, caracterizada por la disminución de la biomasa y actividad microbiana. El inóculo de vermicompost en purín de cerdo produjo el mismo efecto (aumento inicial y posterior descenso) aunque de menor entidad, probando la existencia de efectos indirectos de las lombrices de tierra en el vermicompostaje. En las deyecciones de las lombrices aumentó la biomasa microbiana y la actividad disminuyó, lo que sugiere la existencia de factores implicados en las relaciones lombrices-microorganismos más allá de la simple digestión y los procesos asociados a la inoculación de las deyecciones en el sustrato.
\end{abstract}

Palabras clave: Eisenia fetida, purín de cerdo, biomasa microbiana, actividad microbiana, actividad enzimática.

Aira, M. \& J. Domínguez. 2010. Earthworms and microorganisms: disentangling the black box of vermicomposting. Acta Zoológica Mexicana (n.s.), Número Especial 2: 385-395.

ABSTRACT. Earthworms affect microorganisms both directly (enhancing or reducing their populations by digesting the substrate) and indirectly (derived effects, such as the production of castings in the fresh substrate). To separate these effects we performed three experiments. In the first, we studied the whole process of vermicomposting, using Eisenia fetida and pig manure in all stage, from fresh residue to vermicompost. In the second experiment, we inoculated pig manure with vermicompost to study the indirect effects of earthworms on decomposition. In the third, we analysed the fresh casts to study direct

Recibido: 16/05/2008; aceptado: 08/01/2010. 
effects of earthworms on microorganisms. Earthworms promoted significant increases in both microbial biomass and activity when they were present in the layers, and in aged layers where earthworm were absent, microbial stabilization occurred, with reduced biomass and activity. Inoculation with vermicompost produced the same increases in microbial biomass and activity, and although these increments were not as high as in presence of earthworms, indirect effects were strong enough to alter the dynamics of organic matter decomposition. In castings, microbial biomass increased, while activity decreased, suggesting the presence of other factors involved in earthworm-microbial relationships, beyond simple digestion and inoculation effects.

Key words: Eisenia fetida, pig manure, microbial biomass, microbial activity, enzymatic activity

\section{INTRODUCCIÓN}

El vermicompostaje se define como un proceso de biooxidación, degradación y estabilización de la materia orgánica, a través de la acción conjunta de algunas lombrices de tierra y microorganismos, mediante el que se obtiene un material final estabilizado, homogéneo, rico en nutrientes y de granulometría fina denominado vermicompost (Domínguez 2004). Las lombrices participan en el proceso realizando diferentes acciones a diferentes niveles espaciales y temporales; entre sus roles más importantes cabe destacar: a) la fragmentación física del sustrato orgánico que aumenta la superficie de ataque para los microorganismos al fragmentarlo; b) la modificación, transporte e inóculo de la microflora presente en el residuo (Lores et al. 2006, Aira et al. 2007); y c) la aireación del sustrato a través de sus actividades de excavación y deyección. De hecho, las transformaciones de las propiedades físico-químicas y bioquímicas de los sustratos orgánicos (Domínguez 2004) y la rapidez con que estas transformaciones ocurren (Aira et al. 2002) hacen del proceso de vermicompostaje un buen sistema para estudiar las relaciones entre las lombrices de tierra epigeas y los microorganismos (Aira et al. 2006, 2007ab). No obstante, la mayoría de los resultados publicados hacen referencia únicamente a las diferencias en parámetros químicos y microbiológicos entre el residuo fresco y el vermicompost.

Un problema importante para determinar los efectos de las lombrices de tierra en el proceso de vermicompostaje es que muchas de las funciones que realizan ocurren en las mismas coordenadas temporales y espaciales, lo que dificulta la separación de los efectos directos, aquellos relacionados con la propia actividad directa de las lombrices en el sistema, como son la digestión de la materia orgánica y los microorganismos, de los indirectos (Fig. 1). Los efectos indirectos se definen como los derivados de los efectos directos, i.e. cómo la modificación de la materia orgánica del sustrato afecta los microorganismos, o como las comunidades microbianas aparecidas en las deyecciones modifican los procesos de descomposición (Fig. 1). Para estudiar y cuantificar los efectos directos e indirectos en el proceso de vermicompostaje realizamos tres experimentos con la lombriz de tierra Eisenia fetida y purín de cerdo como sustrato. En el primer experimento estudiamos el proceso de vermicompostaje, desde el residuo fresco hasta el vermicompost con todos sus pasos intermedios; en 


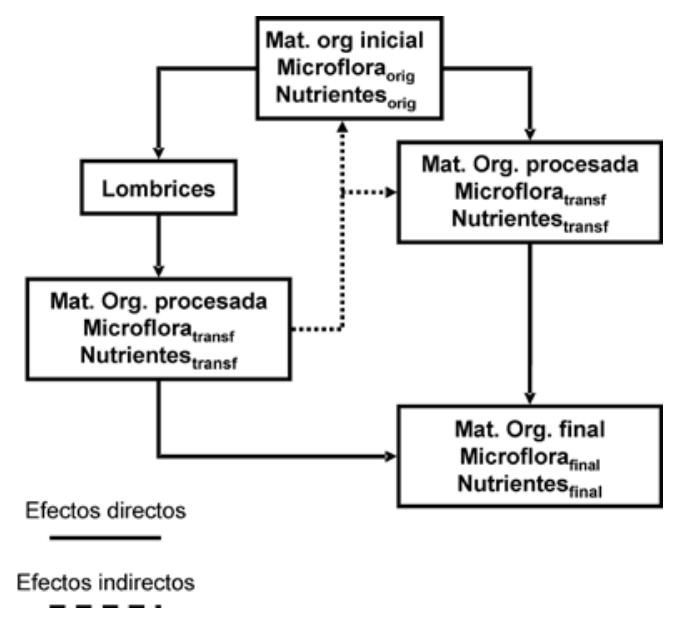

Figura 1. Efectos directos e indirectos de las lombrices durante el vermicompostaje. Las líneas sólidas representan efectos directos y las punteadas efectos indirectos.

este experimento no se pueden separar los efectos directos de las lombrices de tierra de los efectos indirectos. En el segundo experimento inoculamos los vermicomposts producidos por diferentes especies de lombrices de tierra en purín de cerdo para estudiar los efectos indirectos, esto es, si la presencia de un sustrato modificado por la lombriz de tierra tiene los mismos efectos que la presencia de la propia lombriz de tierra. En el tercer experimento estudiamos las deyecciones frescas de la lombriz de tierra E. fetida como estimación de los efectos directos, esto es los debidos a los procesos digestivos de la lombriz.

\section{Diseños experimentales}

\section{MATERIAL Y MÉTODOS}

En el experimento de vermicompostaje utilizamos vermireactores de alimentación continua de diseño propio. Para realizar el experimento montamos 6 reactores, 3 de ellos sin lombrices (control) y 3 con una población inicial de 500 lombrices, con una biomasa de $90 \pm 10 \mathrm{~g}$. Al inicio del experimento cada vermireactor estaba compuesto de un modulo relleno de vermicompost, donde se introdujeron las lombrices, y un modulo con $1.5 \mathrm{~kg}$ de purín. Se añadieron nuevos módulos con $1.5 \mathrm{~kg}$ de purín fresco cuando las lombrices lo necesitaron (Aira et al. 2006, 2007a). Este diseño nos permitió conocer la edad del sustrato de cada módulo. Al final del experimento los reactores estaban compuestos de 12 módulos, con edades de 2, 4, 7, 8, 11, 18, 21, $25,27,29,33$ y 36 semanas. En el muestreo recogimos muestras compuestas (5 submuestras al azar por módulo). Se analizaron el C-biomasa microbiana, el ergosterol, 
la respiración basal e inducida, y las actividades enzimáticas $\beta$-glucosidasa, celulasa, fosfatasa y proteasa.

Para el experimento de inoculación de vermicompost utilizamos microcosmos consistentes en tarrinas de plástico de $200 \mathrm{ml}$ cubiertas con tapas perforadas. Establecimos tres tratamientos de inoculación de vermicompost (0,2.5 y 10\%) para cada uno de los tres vermicomposts utilizados (Eisenia andrei, E. fetida y Eudrilus eugeniae). Los tres vermicomposts utilizados se obtuvieron en el laboratorio a partir de cultivos de las tres especies de lombrices de tierra. El vermicompost se mezcló con el purín de cerdo hasta conseguir una distribución homogénea; las réplicas sin inocular ( $0 \%$ de vermicompost) recibieron el mismo tratamiento. Montamos 5 réplicas por tratamiento, i.e. tipo de vermicompost, porcentaje de inoculación y tiempo. Los microcosmos se mantuvieron a $20^{\circ} \mathrm{C}$ en una cámara de incubación. A los 15,30 y 60 días se muestrearon destructivamente un subconjunto de muestras para cada tipo de vermicompost y porcentaje de inoculación $(n=5)$. Los análisis realizados fueron los mismos que en el experimento de vermicompostaje.

Para analizar las deyecciones, preparamos contenedores de plástico $(n=15)$ de cinco litros de capacidad, en los que dispusimos vermicompost hasta alcanzar $3 / 4$ de su capacidad. En cada contenedor establecimos una población de la lombriz de tierra $E$. fetida en alta densidad para conseguir una cantidad elevada de deyecciones; el purín de cerdo (200 gramos peso fresco) se depositaba sobre el vermicompost. Los contenedores se revisaron a diario para recoger las deyecciones frescas (muestra compuesta en cada contenedor) y analizar el C-biomasa bacteriana, ergosterol, respiración basal, y las enzimas dehidrogenasa, $\beta$-glucosidasa y fosfatasa básica.

\section{Métodos analíticos}

El ergosterol se analizó con una extracción asistida por microondas y posterior detección por HPLC (Young 1995). El C-biomasa microbiana se determinó con el método de fumigación-extracción (Vance et al. 1987), con un factor de eficiencia de extracción para el C microbiano de 2.64. Las respiraciones basal e inducida se determinaron midiendo la tasa de producción de $\mathrm{CO}_{2}$ después de 6 y 12 horas de incubación con trampas de $\mathrm{NaOH}$. A las muestras para la respiración inducida se les añadieron 0.75 $\mathrm{mL}$ de una solución de glucosa de $100 \mathrm{mg} \mathrm{g}^{-1}$ (Aira et al. 2002, Anderson 1982). La actividad de la $\beta$-glucosidasa se determinó midiendo la liberación de $\rho$-nitrofenol tras la incubación de las muestras con $\rho$-nitrofenol glucósido $(0.025 \mathrm{M})$ durante 1 hora (Eivazi \& Tabatabai 1988). La actividad de la celulasa se determinó analizando los azúcares reducidos después de la incubación con carboximetil celulosa durante 24 horas (Schinner \& Von Mersi 1990). La actividad de la fosfatasa alcalina se determinó midiendo la liberación de $\rho$-nitrofenol tras la incubación de las muestras con $\rho$-nitrofenol fosfato $(0.025 \mathrm{M})$ durante 1 hora (Eivazi \& Tabatabai 1977). La actividad 
de la proteasa se determinó analizando los amino ácidos liberados después de incubar las muestras con caseinato sódico durante 2 horas (Ladd \& Buttler 1972).

\section{Análisis estadísticos}

Los datos de los experimentos de vermicompostaje e inoculación se analizaron con un análisis de componentes principales, para resumir el comportamiento general de las muestras en función de las distintas variables analizadas. Los datos del experimento de deyecciones frescas se analizaron con una t de Student. Todos los análisis se hicieron con el paquete estadístico SPSS 11.5.

\section{RESULTADOS}

\section{Experimento vermicompostaje (efectos directos e indirectos)}

Al final del experimento de vermicompostaje ( 36 semanas) la población de lombrices era 5 veces mayor en número $(2800 \pm 200)$ y 8 veces mayor en biomasa $(700 \pm 30$ g). Las lombrices se localizaron principalmente en las capas más jóvenes (2-18 semanas de edad), siendo más abundantes en las dos capas más jóvenes ( 2 y 4 semanas de edad) (Aira et al. 2006, 2007a). La actividad de las lombrices en las capas más jóvenes (2-11 semanas de edad) produjo aumentos en el C-biomasa microbiana, ergosterol, respiración basal e inducida y las cuatro enzimas analizadas ( $\beta$-glucosidasa, celulasa, fosfatasa básica y proteasa), aunque el aumento fue bastante variable entre las cinco capas, esto es, los mayores picos de biomasa y actividad se registraron en las capas de 2 y 4 semanas de edad, lo que desplazó las muestras al cuadrante superior derecho de la figura (Fig. 2). En las muestras de mayor edad (18-36 semanas de edad), este efecto revirtió, con marcados descensos en todas las variables, muy similares en todas las capas; en el caso de los vermireactores sin lombrices apenas hubo variación en los parámetros microbianos analizados, lo que se reflejó en un mayor grado de agrupación de las muestras en el análisis de componentes principales al desplazarse al cuadrante inferior derecho (Fig. 2).

\section{Experimento inoculación vermicompost (efectos indirectos)}

En el experimento de inoculación de vermicompost las muestras se agruparon en función del tiempo de incubación (15, 30 y 60 días) (Fig. 3). La evolución de las muestras inoculadas $(2,5 \%$ y $10 \%$ de inóculo) siguió un patrón similar al mostrado por las muestras control ( $0 \%$ de inóculo) en los tres tiempos de incubación, aunque hubo pequeñas diferencias dependiendo del tipo de vermicompost utilizado para la inoculación. Así, en las muestras inoculadas con un 2,5\% de vermicompost tras 15 días de incubación aumentó la actividad enzimática (proteasa, celulasa y fosfatasa básica) y respiración basal (Fig.3). Tras 30 días, en las muestras inoculadas con vermicompost de E. eugeniae y E. andrei disminuyó la respiración basal y aumentó el 
Aira \& Domínguez: Relaciones microbios-lombrices de tierra en el vermicompostaje

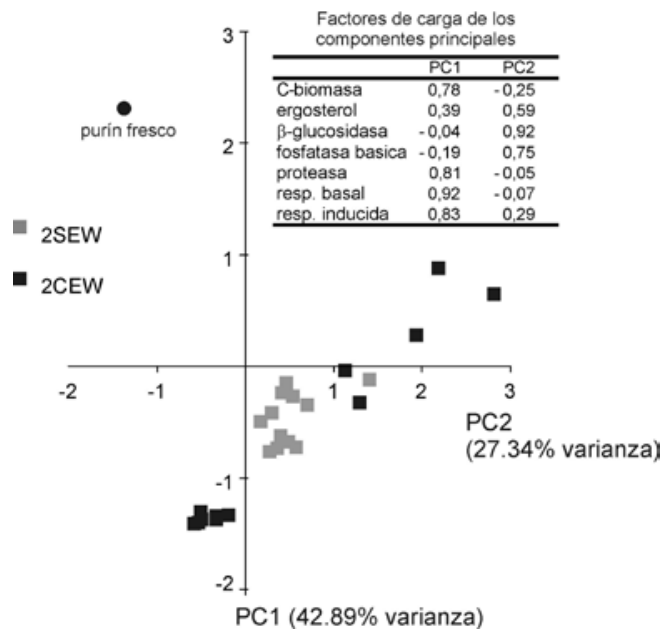

Figura 2. Análisis de componentes principales con las diferentes variables de biomasa y actividad microbiana. Se representa la evolución a lo largo del tiempo de las muestras sin lombrices (gris, 2SEW) y con lombrices (negro, 2CEW).

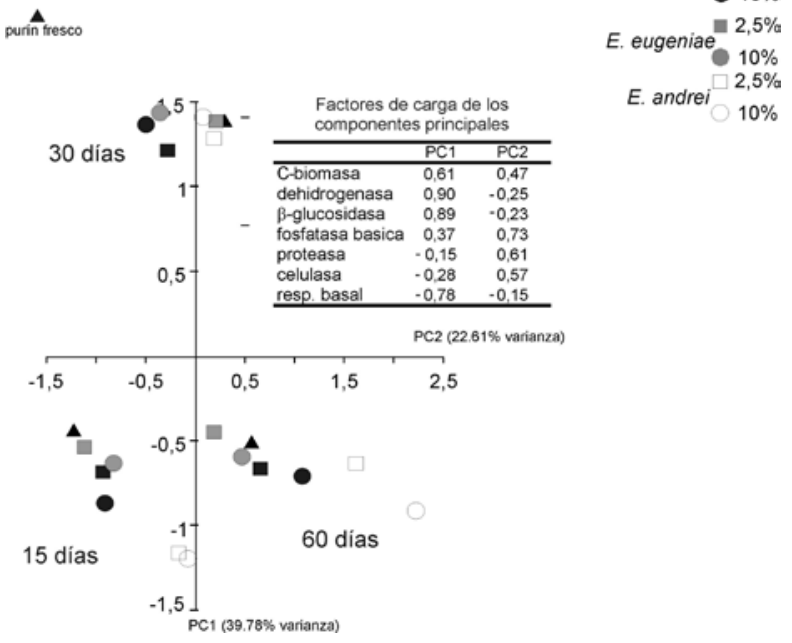

Figura 3. Análisis de componentes principales con las diferentes variables de biomasa y actividad microbiana. Se representa la evolución a lo largo del tiempo de las muestras inoculadas con los vermicompost de Eudrilus eugeniae (gris), Eisenia fetida (negro) y Eisenia andrei (blanco), al 2,5\% (cuadrados), 10\% (círculos) y sin inocular (control, triángulos). 
C-biomasa microbiana y las actividades $\beta$-glucosidasa y dehidrogenasa; en las muestras inoculadas con vermicompost de E. fetida aumentó la actividad enzimática (proteasa, celulasa y fosfatasa básica). Tras 60 días aumentó la respiración basal y declinó la actividad de las enzimas $\beta$-glucosidasa y dehidrogenasa. Las muestras inoculadas con los tres tipos de vermicompost tienen valores similares en estas tres variables pero se separan perfectamente porque los incrementos de actividad enzimática (proteasa, celulasa y fosfatasa) y C-biomasa microbiana, se ordenaron de mayor a menor entidad E. andrei $>$ E. fetida $>$ E. eugeniae. Las muestras con un $10 \%$ de inóculo de vermicompost de $E$. andrei mostraron un patrón idéntico a las muestras inoculadas con un $2.5 \%$ diferenciándose claramente en los valores del día 60 , con un mayor desplazamiento en la componente 2 , caracterizado por una mayor biomasa microbiana y actividad de las enzimas proteasa, celulasa y fosfatasa básica. En las muestras inoculadas con un $10 \%$ de vermicompost las diferencias entre distintos vermicompost aparecieron al día 60, que estuvo caracterizado por un aumento de la actividad de las enzimas proteasa, celulasa y fosfatasa básica, que fue mayor en las muestras inoculadas con vermicompost de $E$. andrei que en las muestras inoculadas con vermicompost de E. eugeniae y E. fetida.

\section{Experimento deyecciones (efectos directos)}

El C-biomasa microbiana aumentó de manera significativa tras el paso por el intestino de la lombriz de tierra E. fetida (Cuadro I; t Student $=-3.22, \mathrm{gl}=28, p<0.01$ ); sin embargo no se encontraron diferencias en el contenido de ergosterol entre el purín de cerdo y las deyecciones frescas (Cuadro I; t Student $=-1.88, \mathrm{gl}=28, p=0.07$ ). $\mathrm{La}$ respiración basal fue significativamente menor en las deyecciones que en el purín de cerdo (Cuadro I; t Student $=2.98, \mathrm{gl}=28, p<0.01$ ), al contrario que la actividad de la enzima dehidrogenasa donde no se encontraron diferencias (Cuadro I; $\mathrm{t}$ Student $=$ $1.26, \mathrm{gl}=28, p=0.22$ ). La actividad de la enzima $\beta$-glucosidasa disminuyó de mane-

Cuadro I. Parámetros microbiológicos en el purín de cerdo y las deyecciones de la lombriz de tierra Eisenia fetida. (Media $\pm \mathrm{ES}, n=15)(\mathrm{ps}=$ peso seco, $\mathrm{mo}=$ materia orgánica, TPF = trifenil formazán, $\mathrm{PNP}=\mathrm{p}$-nitrofenol).

\begin{tabular}{lcc}
\hline & Purín de cerdo & Deyecciones \\
\hline C-biomasa microbiana $\left(\mu \mathrm{g} \mathrm{g}^{-1} \mathrm{ps}\right)$ & $14070 \pm 550$ & $16580 \pm 540^{*}$ \\
Ergosterol $\left(\mu \mathrm{g} \mathrm{g}^{-1} \mathrm{ps}\right)$ & $0.47 \pm 0,07$ & $0.60 \pm 0,02$ \\
Respiración basal $\left(\mu \mathrm{g} \mathrm{CO}_{2} \mathrm{~g}^{-1} \mathrm{mo} \mathrm{h}^{-1}\right)$ & $810 \pm 15$ & $745 \pm 14^{*}$ \\
Dehidrogenasa $\left(\mu \mathrm{g} \mathrm{TPF} \mathrm{g}{ }^{-1}{\left.\mathrm{ps} 24 \mathrm{~h}^{-1}\right)} \mathrm{g}_{\text {-glucosidasa }(\mu \mathrm{g} \mathrm{PNP} \mathrm{g}} \mathrm{ps}^{-1}\right.$ & $13110 \pm 520$ & $12170 \pm 520$ \\
Fosfatasa básica $\left(\mu \mathrm{g} \mathrm{PNP} \mathrm{g} \mathrm{gs}^{-1}\right)$ & $1360 \pm 60$ & $930 \pm 20^{*}$ \\
\hline
\end{tabular}

*diferencias significativas a $p<0.01$. 
ra significativa tras el paso por el intestino de la lombriz E. fetida (Cuadro I; t Student $=6.65, \mathrm{gl}=28, p<0.01)$, mientras que la actividad de la fosfatasa básica aumentó (Cuadro I; t Student $=-14.48, \mathrm{gl}=28, p<0.001)$.

\section{DISCUSIÓN}

Los resultados del experimento de vermicompostaje muestran que la acción de las lombrices de tierra dividió el proceso de vermicompostaje en dos zonas: la primera o zona activa situada en las capas más jóvenes, donde se observaron las mayores densidades de lombrices de tierra y se registraron aumentos en todas las variables microbianas, tanto de biomasa como de actividad; y la segunda o zona de maduración, compuesta por las capas de mayor edad y caracterizada por la ausencia de lombrices de tierra y la estabilización del sustrato debida a la baja biomasa y actividad microbiana. De hecho, en las capas jóvenes tanto la biomasa como la actividad microbiana mostraron valores hasta 4 veces mayores que las capas correspondientes en los vermireactores sin lombrices (Aira et al. 2006, 2007a). Estos resultados contradicen el hecho generalmente aceptado de que los microorganismos forman parte de la dieta de las lombrices de tierra (Edwards 2004), especialmente los hongos (Dash et al. 1986), que las lombrices de tierra pueden ingerir selectivamente y digerir (Moody et al. 1995, Schönholzer et al. 1999) ya que encontramos que el ergosterol (biomarcador de hongos) también aumentó su concentración en las capas jóvenes, incremento que estuvo asociado a una mejor descomposición de la celulosa (Aira et al. 2006). Nuestros datos sugieren que durante los primeros estadios del vermicompostaje las lombrices y los microorganismos establecen una relación cercana al mutualismo (Brown et al. 2000), aunque en este caso ocurriría fuera del intestino. Este mecanismo sería similar a los procesos de enriquecimiento de nutrientes descritos por Devliegher \& Verstraete (1995), pero en este caso E. fetida modificaría la estructura del sustrato incrementando la disponibilidad de nutrientes gracias a la producción de moco, que además tienen una biomasa microbiana mayor que el purín de cerdo, lo que estimularía la aparición de una microflora más activa y especializada en la descomposición (Aira et al. 2007a).

En el experimento de inoculación encontramos que los efectos indirectos de las lombrices de tierra modificaron la evolución de la biomasa y actividad microbiana con respecto a lo observado en el purín de cerdo sin inocular; esta respuesta dependió del tipo de vermicompost y de la dosis de inoculación (Fig. 3). Estos cambios fueron en la misma dirección (aumento inicial y posterior descenso) que los observados en el experimento del vermicompostaje, aunque de menor entidad. Las hipótesis más plausibles para este efecto son: (i) que el inóculo aporte más biomasa microbiana, enzimas extracelulares o nutrientes; y (ii) el aporte de una microflora distinta y más especializada que la del purín de cerdo. Ninguno de los vermicomposts presentó valo- 
res más altos que el purín de cerdo en ninguna variable analizada salvo para la enzima celulasa, por lo que la primera hipótesis puede descartarse. Sin embargo se sabe que los vermicomposts tienen una microflora distinta de la del residuo fresco, que depende más que del residuo de partida que de la especie de lombriz utilizada (Lores et al. 2006). Además, la diversidad metabólica de los microorganismos presentes en los vermicompost es mayor que la del purín de cerdo, lo que mejora las tasas de descomposición (Aira et al. 2007a) por lo que la segunda hipótesis parece la más plausible.

Los resultados del experimento de las deyecciones (aumento biomasa y descenso actividad microbiana) indican que los efectos directos de la lombriz E. fetida producen cambios que influenciarán la dinámica de la degradación de la materia orgánica al incrementar la biomasa pero no la actividad microbiana; la actividad microbiana disminuyó probablemente por las reducciones a nivel de $\mathrm{C}$ y $\mathrm{N}$ orgánico disuelto encontradas en las deyecciones con respecto al purín de cerdo (Aira \& Domínguez 2009). Sin embargo, las deyecciones también estuvieron caracterizadas por un incremento del potencial enzimático que podría conllevar una mejor degradación del purín de cerdo. El aumento de la biomasa microbiana contradice de nuevo el hecho de que los microbios forman parte de la dieta de las lombrices de tierra (Edwards 2004, Moody et al. 1995).

Los resultados del trabajo se pueden resumir en la Fig. 4, donde se representan las dos partes del proceso de vermicompostaje en función de la presencia de las lombrices de tierra. Esto es el resultado de la acción conjunta de efectos directos e indirectos de la lombriz de tierra E. fetida. La posible causa del aumento inicial teniendo en cuenta los valores de biomasa y actividad microbiana en las deyecciones (aumento y disminución respectivamente), podría ser el aporte de nutrientes como el carbono orgánico en disolución (Aira et al. 2007a), limitante para los microorganismos y por

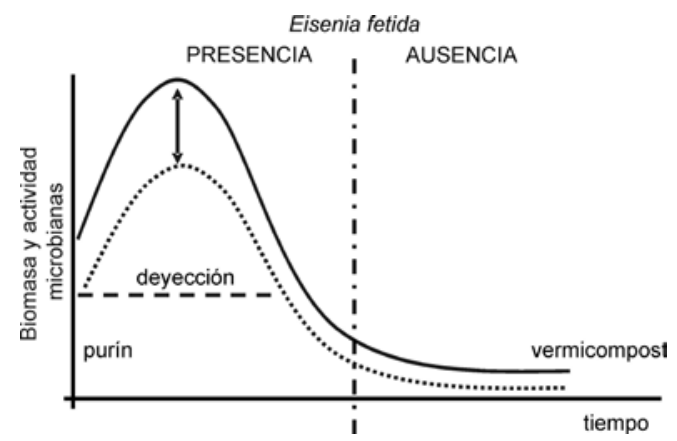

Figura 4. Efectos directos e indirectos de las lombrices de tierra durante el vermicompostaje de purín de cerdo. La línea sólida representa los resultados del experimento de vermicompostaje y la línea discontinua la del experimento de inoculación. La flecha indica la separación entre los efectos indirectos y la suma de directos e indirectos. 
el que compiten con las lombrices (Tiunov \& Scheu 2004), o cambios en la estructura del sustrato que favorezcan la disponibilidad de nuevas fuentes de alimento para los microorganismos, o la aparición de una nueva microflora más especializada (Lores et al. 2006, Aira et al. 2007a). Al comparar los efectos derivados de la inoculación de vermicomposts con el vermicompostaje probamos que la microflora sigue una dinámica similar pero los efectos son de menor entidad, lo que sugiere el importante papel de las lombrices de tierra durante el proceso. Las posibles causas podrían ser un aporte insuficiente de nutrientes de los vermicomposts utilizados, o que la microflora del vermicompost de E. fetida fuera escasa y estuviera ya especializada en metabolizar otro tipo de sustratos, ya que las muestras inoculadas con vermicompost de $E$. andrei mostraron valores mayores de biomasa y actividad microbiana.

\section{AGRADECIMIENTOS}

Manuel Aira está financiado por el programa de investigación Isidro Parga Pondal de la Xunta de Galicia. Este trabajo ha sido financiado por la Xunta de Galicia (proyecto 07MRU023383PR) y el Ministerio de Ciencia e Innovación (proyecto CTM200908477).

\section{LITERATURA CITADA}

Aira, M., F. Monroy, J. Domínguez \& S. Mato. 2002. How earthworm density affects microbial biomass and activity in pig manure. European Journal of Soil Biology. 38: 7-10.

Aira, M., F. Monroy \& J. Domínguez. 2006. Eisenia fetida (Oligochaeta, Lumbricidae) activates fungal growth, triggering cellulose decomposition during vermicomposting. Microbial Ecology. 52: 738-746.

Aira, M., F. Monroy \& J. Domínguez. 2007a. Eisenia fetida (Oligochaeta: Lumbricidae) modifies the structure and physiological capabilities of microbial communities improving carbon mineralization during vermicomposting of pig manure. Microbial Ecology. 54: 662-671.

Aira, M., F. Monroy \& J. Domínguez. 2007b. Microbial biomass governs enzyme activity decay during aging of worm-worked substrates through vermicomposting. Journal of Environmental Quality. 36: 448-452.

Aira, M. \& J. Domínguez. 2009. Microbial and nutrient stabilization of two animal manures after the transit through the gut of the earthworm Eisenia fetida (Savigny, 1826). Journal of Hazardous Materials. 161: 1234-1238.

Anderson, J. P. E. 1982. Soil respiration. Pp 831-871. In: A. L. Page and R. H. Miller (Eds.). Methods of soil analysis, Part 2. Chemical and microbiological properties, $2^{\text {nd }}$ Ed. Agronomy Monograph No. 9, ASA-SSSA, Madison.

Brown, G. G., I. Barois \& P. Lavelle. 2000. Regulation of soil organic matter dynamics and microbial activity in the drilosphere and the role of interactions with other edaphic functional domains. European Journal of Soil Biology. 36: 177-198.

Brown, G. G. \& B. M. Doube. 2004. Functional interactions between earthworms, microorganisms, organic matter, and plants. Pp. 213-241. In: C. A. Edwards (Ed.). Earthworm ecology, $2^{\text {nd }}$ Ed. CRC Press, Boca Raton. 
Dash, H. K., B. N. Beura \& M. C. Dash. 1986. Gut load, transit time, gut microflora, and turnover of soil, plant, and fungal material by some tropical earthworms. Pedobiologia. 29: 13-20.

Devliegher, W. \& W. Verstraete. 1995. Lumbricus terrestris in a soil core experiment: nutrient-enrichment processes (NEP) and gut-associated processes (GAP) and their effect on microbial biomass and microbial activity. Soil Biology \& Biochemistry. 27: 1573-1580.

Domínguez, J. 2004. State of the art and new perspectives on vermicomposting research. Pp. 401-424. In: C.A. Edwards (Ed.). Earthworm ecology, $2^{\text {nd }}$ Ed. CRC Press, Boca Raton.

Domínguez, J., P. J. Bohlen \& R. W. Parmelee. 2004. Earthworms increase nitrogen leaching to greater soil depths in row crop agroecosystems. Ecosystems. 7: 672-685.

Edwards, C. A. 2004. Earthworm ecology, $2^{\text {nd }}$ Ed. CRC Press, Boca Raton.

Eivazi, F. \& M. A. Tabatabai. 1988. Glucosidases and galactosidases in soils. Soil Biology \& Biochemistry. 20: 601-606.

Eivazi, F. \& M. A. Tabatabai. 1977. Phosphatases in soils. Soil Biology \& Biochemistry. 9: 167-172.

Ladd, J. N. \& J. H. A. Butler. 1972. Short-term assays of soil proteolytic enzyme activities using proteins and dipeptide derivatives as substrates. Soil Biology \& Biochemistry. 4: 19-30.

Lores, M., M. Gomez-Brandon, D. Perez-Diaz \& J. Domínguez. 2006. Using FAME profiles for the characterization of animal wastes and vermicomposts. Soil Biology \& Biochemistry. 38: 29932996.

Moody, S. A., M. J. I. Briones, T. G. Pierce \& J. Dighton. 1995. Selective consumption of decomposing wheat straw by earthworms. Soil Biology \& Biochemistry. 28: 533-537.

Schinner, F. \& W. von Mersi. 1990. Xylanase-, CM-cellulase- and invertase activity in soil: an improved method. Soil Biology \& Biochemistry. 22: 511-515.

Schönholzer, F., D. Hahn \& J. Zeyer. 1999. Origins and fate of fungi and bacteria in the gut of Lumbricus terrestris L. studied by image analysis. FEMS Microbiology Ecology. 28: 235-248.

Tiunov, A. V.\& S. Scheu. 2004. Carbon availability controls the growth of detritivores (Lumbricidae) and their effect on nitrogen mineralization. Oecologia. 138: 83-90.

Vance, E. D., P. C. Brookes \& D. S. Jenkinson. 1987. An extraction method for measuring soil microbial biomass C. Soil Biology \& Biochemistry. 19: 703-707.

Young, J. C. 1995. Microwave-assisted extraction of the fungal metabolite ergosterol and total fatty acids. Journal of Agricultural and Food Chemistry. 43: 2904-2910. 